Didier Perret

Université Rennes 2 (France)

Pascal Plantard

Université Rennes 2 (France)

\section{Pratiques numériques des enseignants en Bretagne pendant le confinement.
Analyse anthropologique des premières données
qualitatives et quantitatives. en Bretagne pendant le confinement.
Analyse anthropologique des premières données
qualitatives et quantitatives. en Bretagne pendant le confinement.
Analyse anthropologique des premières données
qualitatives et quantitatives.}

Digital practices of teachers in Bretagne during the confinement. An anthropological analysis of preliminary qualitative and quantitative data

doi: $10.18162 / f p .2020 .686$

\section{ésumé}

Cet article s'interroge sur les dimensions personnelles et collectives des parcours d'appropriation des technologies numériques par les enseignants du second degré en se focalisant sur leurs pratiques numériques durant le confinement. Une approche qualitative par entretiens semi-directifs vient compléter les données quantitatives recueillies à partir de quatre enquêtes réalisées auprès des enseignants, des élèves et des familles de 2018 à 2020. Les résultats présentent différentes dynamiques d'appropriation et s'attardent aux relations entre les différents acteurs, enseignants, élèves et parents. Ils interrogent également l'organisation des dispositifs d'accompagnement et de formation des enseignants..

\section{Mots-clés}

Anthropologie des usages, parcours d'appropriation, enseignants, technologies numériques, pratiques numériques.

Abstract

This article investigates the individual and collective dimensions of pathways of digital technology appropriation by secondary school teachers, with a focus on digital practices during the confinement. A qualitative approach using semi-directed interviews was combined with quantitative data gathered from four surveys of teachers, students, and families from 2018 to 2020 . The results reveal various dynamics of appropriation and highlight issues in the relationships between the various actors: teachers, students, and parents. They also call for improved support and training programs for teachers..

Keywords

Anthropology of uses, appropriation pathways, teachers, digital technology, digital practices

\section{Introduction}

Avec le réseau M@rsouin de recherche sur les usages des technologies numériques, l'équipe de recherche du Centre de Recherche sur l'Éducation, les Apprentissages et la Didactique (CREAD) étudie depuis 18 ans les pratiques numériques éducatives des élèves, des familles et des enseignants en Bretagne. Capitalisant sur les résultats d'un ensemble de projets de recherche situés en Bretagne (dont les ANR INEDUC 2012-2015, CAPACITY 2014-2017 et « Technographie de collège connecté » 2013-2015), nos travaux actuels s'inscrivent dans le projet Interactions Digitales pour l'Éducation et l'Enseignement ${ }^{1}$ (IDÉE, 2017-2021) qui s'intéresse aux pratiques enseignantes et à la réduction des inégalités de parcours éducatifs. Dans le cadre de ce projet IDÉE, nous suivons depuis 2017 des cohortes de 3500 élèves et familles en menant des enquêtes quantitatives passées dans les classes et envoyées aux familles par l'établissement scolaire. Pour les enseignants, le choix a été fait d'adopter un questionnaire en ligne déployé en janvier 2020 et ayant recueilli 1000 répondants, articulé avec le dispositif de soutien à l'innovation pédagogique et numérique Interactik soutenu par la région Bretagne et l'académie de Rennes. Le volet Appropriatik du programme IDÉE se focalise sur la mise en place d'un dispositif particulier d'accompagnement et de formation continue des enseignants, les coopératives pédagogiques numériques $(\mathrm{CPN})$. À partir du questionnement institutionnel sur la formation continue des enseignants (rapport de l'Inspection générale de l'Éducation nationale, septembre 2018), il est nécessaire d'interroger les difficultés que rencontrent les enseignants avec les dispositifs classiques de formations académiques dans le cadre de l'appropriation des technologies. Au travers de notre recherche, nous nous sommes intéressés à la place que peuvent prendre les 
acteurs humains, en présence ou à distance, comme facilitateurs dans l'appropriation des technologies numériques. Complétées par de nombreuses observations qualitatives, ces données quantitatives vont nous permettre d'avoir une vision assez précise des usages pédagogiques des technologies numériques tant par les élèves que par les enseignants de Bretagne avant le confinement.

L'expérience du confinement est intense et massive. C'est un " fait social total ", pour reprendre l'expression de Marcel Mauss. Notre société et notre école en sortiront profondément modifiées. Le recours à l'enseignement à distance ne va pas de soi et nous souhaitons conserver des traces scientifiques de cette période pour participer à cette évolution dans le sens de la prise en compte des inégalités éducatives. Nous avons actualisé et redéployé notre questionnaire dès le 8 avril 2020 pour prendre en compte la situation de confinement. Ce questionnaire est parfaitement anonyme et respecte le règlement général sur la protection des données (RGPD). Mi-juillet 2020, nous avons recueilli 524 réponses exploitables. Il comprend de nombreuses plages d'expression libre que nous n'avons pas encore analysées; cependant, nous avons pu effectuer une comparaison des premiers tris croisés des deux questionnaires qui nous permettent d'avancer prudemment quelques résultats provisoires pour cet article sous la forme de tendances et non de chiffres précis qui viendront ultérieurement.

\section{Contexte, ancrages théoriques et objectifs}

Plusieurs auteurs s'accordent sur l'importance du numérique dans l'éducation : pour Proulx (2005) :

Internet [et le numérique dans son ensemble ?] peut produire un effet de levier dans la réorganisation sociale et économique des sociétés industrielles. L'avènement d'Internet se situe dans un contexte socio-historique plus vaste que le seul développement des machines à communiquer. (Proulx, 2005, p. 4)

Internet et l'ensemble des services et des applications associées viennent modifier sept domaines de la vie courante : « la communication, la politique, la sociabilité, l'identité et la subjectivité, la création culturelle [à laquelle se rattachent les pratiques en éducation], le travail humain et l'économie » (Proulx, 2005). La question se pose au sujet des compétences nécessaires à acquérir, pour les citoyens, afin de maîtriser ces changements de la société. Selon Cottier et Burban (2016) :

Les technologies numériques constituent un ensemble conséquent d'artéfacts dont il serait illusoire de dresser un tableau exhaustif, car il s'agit de technologies nombreuses et hybridées [...] le substantif numérique renvoie quant à lui au fait social, aux dimensions non exclusivement artéfactuelles de ce qu'il serait sans doute plus juste de nommer le fait numérique. (Cottier et Burban, 2016, p. 9)

En reprenant l'essai sociologique sur le don de Marcel Mauss, plusieurs auteurs donnent une envergure pluridimensionnelle aux transformations de la société (technique, politique, économique, culturelle et sociale), une dimension de «fait social total » porteuse de règles et de normes qui s'imposent progressivement aux individus (Plantard, 2014; Cottier et Burban, 2016). C'est pourquoi notre approche des technologies numériques est anthropologique en ce qu'elle définit les usages comme des normes sociales d'usages (De Certeau, 1990) et qu'elle prend en compte la profondeur historique et la dimension symbolique de la construction des usages.

2 - Formation et profession 28(4 hors-série), 2020 
La question de l'appropriation des technologies par les enseignants prend sa source dans les travaux de Proulx et Jouet qui définissent que l'appropriation est un parcours itératif et non un état de fait. Si l'on peut identifier aujourd'hui au moins 19 modèles s'intéressant à l'intégration des technologies numériques en classe (Fiévez, 2017), on déplore que la plupart soient technocentrés. Plantard (2016) présente le parcours d'appropriation d'une technologie numérique en quatre étapes (figure 1) :

La phase amorce renvoie au temps de la découverte et de l'étonnement. Les phases de confiance et de construction participent du temps de comprendre. La phase d'autonomisation marque le temps de l'incorporation, de la réflexivité et du contre-don. L'évolution de la personne dans le processus d'appropriation est modélisée en phases, mais n'est pas linéaire. En fonction de la situation de la personne et de l'instrument, le parcours peut revenir à une phase antérieure, voire reboucler complètement. (Plantard, 2016)

L'entrée dans le parcours se fait soit par un désir propre à l'individu (réduire les inégalités, modifier le fonctionnement des cours, accompagner un projet spécifique ou juste réaliser une activité spécifique avec les élèves), soit par contrainte (norme perçue, obligation des programmes, voir confinement comme cela a été le cas en mars 2020). C'est un processus qui va s'insérer dans un contexte socioculturel spécifique à chacun (Larroze-Marracq, 1999), en se basant sur un modèle pédagogique évolutif, autour de trois pôles : l'axis, c'est-à-dire les valeurs éducatives défendues ; la praxis, le pôle didactique des pratiques et des méthodes, et la psyché, qui est la dimension émotionnelle et psychologique de la pédagogie.

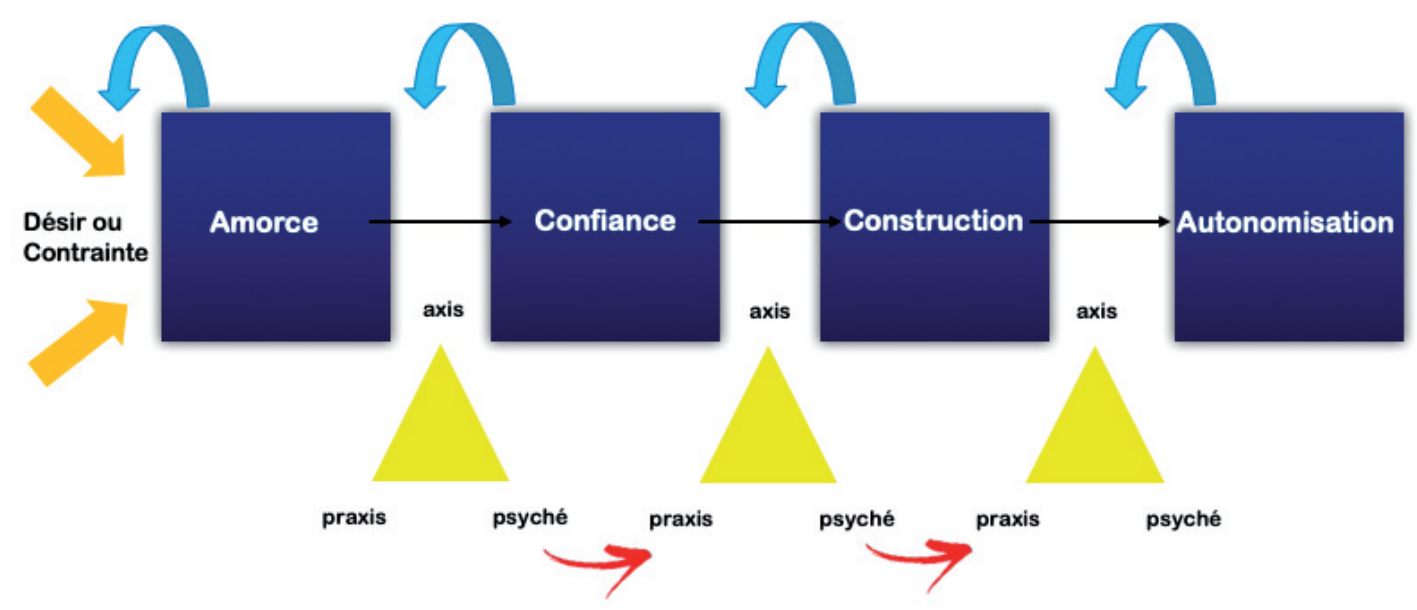

Figure 1

Parcours d'appropriation (Plantard, 2016) - CC-BY-ND

L'appropriation des technologiques est tout d'abord un processus d'acquisition individuel de connaissances et de compétences, des savoirs, des savoir-faire et des habiletés pratiques (Jouët, 2000) qui consiste en une négociation entre l'utilisateur et la technologie mêlée de bricolage (Levi-Strauss, 1962) et de braconnage (Plantard, 2014). Puis l'appropriation se développe dans et autour du collectif. L'activité du sujet se construit au sein d'une communauté qui regroupe les individus ou des sousgroupes partageant le même projet ou le même objectif. Ces communautés spécifiques s'apparentent pour certaines aux communautés de pratique (Wenger, 2005), mais d'autres sont simplement le fait des réseaux personnels des enseignants (Wasserman et Faust, 1994). L'appropriation met en jeu tant 
l'identité personnelle que l'identité sociale de l'individu, d'autant plus que chez les enseignants, les usages numériques professionnels viennent remettre en question l'accomplissement personnel. L'appropriation a lieu entre le moment où se développent les premiers usages et celui où il y a stabilisation de ceuxci qui deviennent alors sociaux. Les difficultés de mise en place des collectifs enseignants sont de plusieurs ordres. Depuis la formation initiale jusqu'à la structuration des équipes, la forme scolaire et les contextes des établissements, il y a tout compte fait peu d'occasions pour construire des compétences de travail collaboratif. Néanmoins, au travers du vécu de différents enseignants de Bretagne pendant le confinement, nous allons évoquer dans cette communication l'influence de processus comme la médiation numérique ${ }^{2}$ dans des environnements capacitants (ex. : les Coopératives Pédagogiques Numériques en Bretagne) ou simplement au sein de petits collectifs informels sur la mise en place des interactions facilitant la mise en œuvre d'activités numériques à distance avec les élèves, pratiques qui nécessitent une certaine appropriation des technologies et du contexte.

\section{Méthodologie}

Les données quantitatives sont issues de quatre campagnes d'enquêtes : les deux premières concernent 3500 élèves de collège et leurs familles avec un suivi de cohorte, un premier passage du questionnaire étant réalisé en 2017-2018 sur les élèves de $5^{\mathrm{e}}$ de 13 collèges puis un second passage auprès de ces mêmes élèves arrivés en $3^{\mathrm{e}}$ et de leurs familles en 2019-2020 (avant le confinement). Les deux autres campagnes concernent les enseignants (premier et second degrés) avec une première enquête en ligne et anonyme qui a recueilli 1000 répondants en 2019 et une seconde, toujours en ligne, à partir d'avril 2020 qui comprend 525 répondants. Les données qualitatives sont issues d'entretiens semi-directifs venant enrichir le dispositif quantitatif (12 enseignants issus de 4 lycées, 7 enseignants issus de 5 collèges, 16 élèves de seconde et première en lycée), complétés par des temps d'observation en classe (une à plusieurs séances d'une heure pour chaque enseignant avec capture vidéo), de rencontres formelles (participation à un groupe de secteur et à des formations) ou informelles (repas, discussions entre deux portes, salle des professeurs...). Pour les entretiens et les observations avec les enseignants, 16 ont été réalisés avant le confinement, 3 ont été faits en juillet 2020 après la période de confinement. Ce choix vise à prendre en considération les dynamiques individuelles et collectives de travail, d'accompagnement et de formation qui influencent, sur un temps long, positivement ou négativement, le degré de mobilisation des instruments numériques (Rabardel, 1995 ; Vergnaud, 1998) par les enseignants dans leurs pratiques pédagogiques quotidiennes. À partir d'une observation participante (Lapassade, 2016) et d'une immersion dans trois lycées et trois collèges (présence sur une journée complète, plusieurs jours de suite chaque mois), nous avons recueilli nos différents matériaux de recherche. La démarche méthodologique s'inscrit dans un processus inductif à partir des entretiens semi-dirigés avec nos enquêtés (1h30 en moyenne). Cela a permis d'identifier les étapes de leurs parcours et surtout d'identifier les personnes présentes dans l'entourage des enseignants (réseau personnel, contacts Twitter, groupes de secteur, communautés de pratiques). De proche en proche, à partir des éléments recueillis sur les premiers entretiens, puis lors des observations participantes en classe et surtout lors des moments d'échange entre enseignants dans différents lieux et différents cadres, d'autres acteurs ont pu être identifiés et contactés. Cette immersion, mais aussi l'observation de formations institutionnelles, de réunions, d'animations au sein des coopératives pédagogiques numériques ont permis de construire un panel étendu de différentes figures d'intervenants sur l'appropriation des technologies numériques.

4 - Formation et profession 28(4 hors-série), 2020 


\section{Résultats et discussion}

\section{Premier tableau}

Dans l'enquête de janvier 2020, on peut identifier un bon quart d'enseignants qui étaient déjà acculturés aux technologies numériques. Depuis le confinement du mois de mars, ils ont pu recréer, même à distance, une dynamique de classe et inventer d'autres façons de travailler. Il y a ensuite la moitié des professeurs qui a tâtonné pour faire au mieux. Au tout début du confinement, certains d'entre eux ont essayé de bien faire en mettant énormément de ressources en ligne, sans vraiment "scénariser ", quitte à noyer les élèves et leurs parents sous les exercices. Après une phase d'adaptation, ils ont dépassé leurs appréhensions, se sont formés à partir de début avril à cet enseignement à distance un peu particulier, pour revenir à quelque chose de plus raisonnable. Ils méritent largement les hommages qui leur ont été rendus, par leur ministre de tutelle comme par les familles. Les problèmes se concentrent sur le quart restant : des enseignants qui, d'ordinaire, ont un usage a minima du numérique en classe - un tableau blanc interactif, des diaporamas... ou pas d'usages du tout. Ces derniers ont rencontré le plus de difficultés. Dans ce groupe, certains s'y sont finalement mis tant bien que mal, avec l'aide de leurs collègues ou de leur famille. D'autres, ultra-minoritaires, mais sur lesquels l'attention se porte depuis quelques semaines, ont décroché malgré eux avec une «casse " psychologique forte (burn-out), des conflits de valeur et un sentiment d'inutilité.

\section{Quelle a été la principale difficulté rencontrée par les professeurs?}

On a vu nombre d'enseignants s'essayer à reproduire, à l'identique, les pratiques scolaires qu'ils ont en classe. Or, cela demande une très forte appropriation des technologies, tant par l'enseignant que par les élèves pour pouvoir fonctionner :

C'est le cas d'Arnaud, enseignant de sciences de la vie et de la terre en collège en centre Bretagne. Enseignant ayant eu un parcours atypique, avec un CAPES obtenu à 29 ans après une période d'activité dans le privé, il est titulaire de son poste depuis plusieurs années maintenant. C'est un enseignant appartenant au groupe des « acculturés aux technologies » cité dans le paragraphe précédent. Il a construit de nombreuses activités avec le numérique permettant aux élèves de participer, de produire et de communiquer, principalement en utilisant une Moodle Box ${ }^{3}$ en local et des tablettes (au moins une tablette pour deux élèves, parfois une tablette par élève suivant les séances). Il utilise aussi dans sa pratique des vidéos, des services tiers comme Canva, Genially ou Learningapps. Le confinement et la mise en place de l'enseignement à distance ne l'ont pas effrayé outre mesure et il va simplement transférer ses activités habituelles réalisées sur la Moodle Box sur le Moodle « espace pédagogique » de l'environnement de travail académique Toutatice. Ainsi, dès les premières semaines, les élèves retrouvent un environnement équivalent à celui qu'ils utilisaient en classe. Des activités de type forum sont menées avec une participation régulière de la plupart des élèves de $3^{\mathrm{e}}$ impliqués. Moins d'une dizaine sur les 110 ne se connecteront pas. Son cours « à distance » est enrichi de vidéos, d'exercices complémentaires avec Learningapps, etc. On voit ici que Arnaud, que l'on peut positionner en phase d'autonomisation, a disposé des ressources nécessaires pour faire face au contexte exceptionnel. Les activités proposées à

distance fonctionnent, non seulement parce qu'elles sont maîtrisées par l'enseignant, mais aussi parce 
que ce sont des activités que les élèves ont déjà réalisées en classe avant le confinement. C'est bien l'adéquation entre ces deux dimensions qu'il faut prendre en compte pour questionner comment enseigner et apprendre aujourd'hui, d'une part le degré d'appropriation de l'enseignant qui va pouvoir adapter ses pratiques au changement de contexte, mais aussi le degré d'appropriation des élèves, leur "culture numérique scolaire " propre qui leur permet de suivre à distance, car étant en terrain connu. Les éléments à disposition pour le moment nont pas pu déterminer si les élèves non impliqués l'ont été du fait d'un problème matériel (connexion, absence de matériel) ou de difficultés de suivi des activités scolaires par les parents. Des entretiens avec les élèves et les familles sont envisagés pour le déterminer. Malgré la réussite des premières activités à distance, Arnaud constate une érosion progressive de l'assiduité des élèves, ce qui interroge la capacité de cette forme d'enseignement à suppléer sur une longue durée l'organisation traditionnelle des cours.

Dans la plupart des cas, les enseignants n'ont pu fonctionner de cette manière. La période inédite que nous venons de vivre, et qui n'est pas encore achevée, a été marquée par un éclatement complet des pratiques pédagogiques, avec, d'un côté, des enseignants tout à fait volontaires pour s'emparer des outils numériques, et, de l'autre, des enseignants pas du tout prêts. Les données qualitatives qui nous reviennent montrent encore une fois l'importance de la formation entre pairs.

Parmi les $50 \%$ d'enseignants qui ont essayé de temporiser et de suppléer au changement du mode d'enseignement, beaucoup se sont appuyés sur les compétences et conseils de leurs collègues déjà acculturés, ou sur ceux des référents « numériques » du premier ou du second degré qui existent dans chaque circonscription ou chaque établissement. Encore faut-il que ceux-ci soient bien repérés comme personnes-ressources par les enseignants, les chefs d'établissement et les académies ...

Dans le cas de Bénédicte, enseignante de sciences physiques en poste dans deux établissements, un lycée général de centre-ville et un collège, c'est justement son réseau personnel qui va lui permettre de suivre plusieurs formations à distance. En effet, dans son établissement, le référent aux ressources et aux usages pédagogiques du numérique ${ }^{4}$ (RRUPN) est aussi formateur académique, membre de l'équipe de la coopérative pédagogique numérique du département. Cet interlocuteur va servir de médiateur pour lui indiquer les liens vers les formations disponibles à distance pour réaliser son projet. En effet, Bénédicte n'a qu'une utilisation très limitée du numérique dans sa pratique pédagogique, Pronote pour saisir les informations sur le suivi des élèves ainsi que Pearltrees pour un dossier partagé avec la classe de seconde dont elle est professeur principal et qui lui permet de suivre les recherches des élèves sur l'orientation. Elle ne communique avec les élèves en dehors des cours que via une adresse courriel dédiée qui ne servait que pour des questions d'organisation d'emploi du temps avant le confinement, soit une ou deux fois dans l'année. Aucun de ses cours n'était disponible en ligne avant le confinement. En classe, les élèves utilisent quelques logiciels disciplinaires en travaux pratiques comme Regressi, elle projette au vidéoprojecteur diaporamas, vidéos et animations flash pour ses cours. Nous pouvons positionner Bénédicte dans la moitié des enseignants qui ont essayé de s'adapter. Par rapport au parcours d'appropriation, elle est entrée dans la phase de confiance. Les technologies numériques commencent à être mises en œuvre, elle a confiance en ses capacités, elle a confiance en ses élèves et commence à appréhender certains outils, mais nétant titulaire dans l'établissement que depuis quatre ans, elle a d'abord dû se concentrer sur "faire d'abord les programmes », créer ses séquences de cours, les modifier en début d'année avec la réforme du lycée. Le développement de ressources en ligne ou d'activités plus élaborées n’était pas sa priorité. L'impératif des activités numériques à distance arrive comme une 
contrainte forte avec le confinement. Or, ses échanges avec le RRUPN de l'établissement, par courriel, lui donnent l'envie de mettre en place des QCM pour ses élèves de lycée sur la plateforme Moodle de l'établissement. À partir de mai, quand sa fille peut reprendre l'école, ce qui lui libère du temps, Bénédicte va ainsi suivre trois formations en visioconférence avec plusieurs formateurs du dispositif académique, une première sur Moodle et la gestion d'un cours sur cette interface, une seconde sur l'activité QCM qui lui apporte ce qu'elle cherche et une troisième sur les modules H5P de Moodle où elle «n’a rien suivi», le sujet étant trop éloigné de ses compétences et de ses besoins. Bénédicte va ainsi créer un cours sur Moodle, y insérer des ressources (PDF, vidéos) et créer plusieurs activités de QCM pour ses élèves. On peut observer une différence ici avec la situation d'Arnaud présentée précédemment : pour Bénédicte, l'activité QCM qu’elle propose aux élèves est une nouveauté et elle va se limiter à cette pratique. Ses élèves n'ont pas pu s'approprier ce processus avant, en classe, ils vont la découvrir à distance. Le premier QCM, noté et donc obligatoire, sera réalisé par 88 sur les 90 élèves de seconde. Toutefois, les 5 QCM suivants, facultatifs, permettant de s'interroger sur les acquis après le visionnage de vidéos, ne seront faits que par 25 élèves au maximum. La phase de confiance est une étape fragile, qui repose sur de nombreux facteurs sociaux et où la présence d'une médiation humaine va être primordiale. D'ailleurs, Bénédicte ne va pas développer d'activités avec Moodle dans son autre établissement, car son seul contact dans l'équipe pédagogique, sa collègue de sciences physiques, n'utilise pas le service et personne ne va pouvoir l'accompagner. Or, dans ce collège, Moodle est déployé depuis 2013, 33 cours sont ouverts... mais ils datent de 2013-2014. N'ayant plus de gestionnaire Moodle actif dans l'équipe pédagogique du collège, le service est tombé en désuétude et ne sera pas utilisé pendant le confinement. Bénédicte disposait des compétences et de l'envie pour mettre en œuvre des activités avec ses élèves de $3^{\text {e }}$, l'outil était présent, mais l'accompagnement humain faisant défaut, elle ne pourra pas aller plus loin pour le moment.

\section{Le confinement des élèves : des tensions sur l'école de la confiance...}

On a beaucoup parlé des "élèves décrocheurs » que la période a particulièrement mis à la peine. On semble aujourd'hui ouvrir les yeux sur les ressentis du confinement et de l'enseignement à distance des «bons » élèves... Avec le déconfinement, le temps est venu de mettre des mots sur cette expérience inédite. La parole des élèves, qu'on a très peu entendus tant qu'ils étaient enfermés chez eux, se libère. Cependant, ce qu'ils ont vu d'hésitations ou d'insuffisances chez leurs enseignants peut nourrir une mise en cause - voire une mise en accusation - qu'ils verbalisent souvent sans filtre. Eux aussi ont entendu dire autour d'eux que « tout se passait au mieux ». Que l'enseignement était assuré « presque comme d'habitude ». Que le CNED - qui était tout sauf un modèle avant la crise - les aiderait à surmonter l'épreuve. Ce discours volontariste, déconnecté de leurs difficultés quotidiennes, a pu être mal vécu. Nous pouvons dire que, pour certains élèves, la confiance à l'égard du système a été bouleversée durant le confinement. Les écoliers ont été en partie épargnés. Les liens plus proches entre les enseignants du $1^{\text {er }}$ degré et les parents ont joué un peu comme un filtre. Les lycéens sont déjà, pour une majorité en tout cas, autonomes dans leur travail et suffisamment mûrs pour trouver un rythme, s'organiser, s'inventer des repères. Recréer un « collectif » via des outils numériques qu'ils maîtrisent - quand ils en sont équipés. Les problèmes se sont concentrés au collège et au lycée professionnel, les niveaux de tous les dangers : les lieux du décrochage, ceux où les inégalités se creusent, et où l'individualisation des enseignements est compliquée. 
Pour Catherine, enseignante d'économie-gestion en lycée professionnel, ce sont justement les difficultés de ses élèves tant du point de vue matériel que scolaire qui vont modeler ses activités pendant le confinement. Dans le parcours d'appropriation, Catherine, comme Arnaud, est en phase d'autonomisation. Elle utilise elle-même de nombreux outils, fait utiliser à ses élèves différents outils durant les cours. Pour elle, utiliser le numérique dans la pédagogie n'est pas seulement faire produire aux élèves un document sur un tableur, c'est plus que cela. Les élèves doivent être en activité, souvent en interaction, et l'utilisation de tel ou tel outil doit participer à la construction d'une culture numérique nécessaire dans leur parcours professionnel. Pourtant, elle constate que ses élèves de filière bac pro commerce sont très peu équipés, souvent simplement d'un smartphone, la plupart n'utilisent pas d'adresse courriel, n'ont pas d'imprimante à la maison, voire pas d'ordinateur. Déjà, avant le confinement, les élèves internes éprouvaient eux aussi des difficultés à travailler le soir, faute d'accès aux salles multimédia. Alors qu'elle est la référente numérique de l'établissement, qu'elle est la gestionnaire de l'interface Moodle pour le lycée, pendant le confinement, elle va limiter les ressources disponibles en ligne à de simples documents à consulter. Ses élèves, disposant de son numéro de téléphone, vont lui transmettre leurs productions par SMS en photographiant le travail réalisé. Catherine limite l'étendue des activités pour permettre aux élèves de pouvoir les réaliser et non par manque de compétence ou manque de connaissances. Ici, enseigner à distance doit prendre en compte aussi les moyens et les capacités des apprenants.

D’autres témoignages en lycée professionnel viennent compléter le tableau présenté avec Catherine. Dans certains cas, le choix des enseignants a été d'aller à l'encontre des directives académiques et d'organiser les échanges avec les élèves sur les réseaux sociaux comme Facebook et Whatsapp. L'important, pour ces enseignants, était de garder le contact avec des élèves en difficulté sociale dont certains ne disposent que d'un accès à internet sur leur smartphone et de manière limitée. Des élèves de lycée professionnel ont décrit qu'ils avaient dû arrêter de travailler pendant plus d'une semaine, car leur forfait $4 \mathrm{G}$ était épuisé et qu'ils ne pouvaient récupérer le travail à faire. Dans ces circonstances, il est inenvisageable de construire un enseignement à distance avec des activités en lignes régulières, c'est-à-dire d'imaginer reproduire à distance le fonctionnement des enseignements habituels. Une telle tentative dans ces conditions ne ferait qu'accentuer les inégalités.

C'est aussi sur le collège et les lycées professionnels que notre vigilance devra être particulière à la rentrée. Les différences de pratiques de ces adolescents viennent renforcer la difficulté pour les enseignants de proposer des activités avec les technologies numériques qui correspondent à leurs besoins tout en correspondant aux programmes.

\section{La «fracture numérique » entre les familles?}

Si le confinement a pu révéler des inégalités entre les familles, il a aussi montré que le concept de "fracture numérique » est une vision caricaturale et idéologique. Personne n'est véritablement « in » ou « out» vis-à-vis du numérique. Résumer le décrochage des élèves de milieu populaire au manque d'équipement numérique, comme on l'entend parfois, c'est faire fi des autres problématiques (économique, sociale, immigration, etc.) qui touchent ces populations et qui expliquent en grande partie leur éloignement de l'institution scolaire. Autrement dit, on pouvait s'attendre à ce qu'ils décrochent. 
Ce qu'a mis en avant de manière plus surprenante la crise, ce sont les fragilités numériques de tous les autres. L'envahissement du numérique via l'école à la maison et le télétravail a mis en difficulté nombre de familles traditionnelles, recomposées, monoparentales, des personnes modestes comme des « cadres sup » ayant fait de bonnes études. De nombreux citadins partis se confiner dans leur maison de campagne se sont aperçus qu'ils ne disposaient pas d'assez de réseau. Si on exclut les jeunes adultes sans enfant des grands centres urbains, tous les Français en ont subi les impacts.

\section{L'importance des dynamiques collectives pour faciliter l'appropriation des technologies}

Dans les trois exemples présentés, ceux d'Arnaud, de Bénédicte et de Catherine, s'il est un point important à compléter, c'est la place des différents interlocuteurs disponibles pour répondre à leurs questionnements durant la phase de confinement, c'est-à-dire la place des dynamiques collectives dans les parcours d'appropriation. Arnaud et Catherine sont tous deux RRUPN, ils disposent d'un compte Twitter qui va les approvisionner en informations, ils font tous deux partie de communautés de pratiques actives sous la forme de groupes de secteur disciplinaires, ils sont en relation régulière avec plusieurs enseignants de leur établissement et d'autres enseignants d'établissements de l'académie. Bien que Catherine mentionne se «sentir seule » dans sa mission de référente numérique, elle va savoir trouver les ressources nécessaires à la modification de ses propres pratiques pendant le confinement auprès de ses contacts, notamment les deux formatrices de la coopérative pédagogique numérique départementale avec qui elle a des échanges réguliers. Elle va aussi proposer des accompagnements à distance, en visioconférence, et des tutoriels, à quelques collègues du secteur tertiaire pour les aider à mettre en œuvre différents outils. Toutefois, elle mentionne la difficulté d'accompagner les autres enseignants, ceux des filières industrielles, voire ceux de l'enseignement général, d'une part par manque de connaissances spécifiques, mais surtout par manque de lien social avec eux, avant le confinement. Pour Arnaud, le constat est sensiblement identique : en tant que RRUPN, il va échanger et accompagner à distance, par courriel, par téléphone ou visioconférence, sept à huit collègues avec qui il a déjà construit une relation régulière avant le confinement. L'équipe de collègues de son collège fonctionne comme une autre communauté de pratiques où chaque enseignant va "visiter » la classe des autres pour découvrir comment il ou elle met en œuvre telle ou telle activité avec les élèves. La période de confinement va permettre un renforcement des échanges à distance au sein de ce groupe, mais Arnaud déplore ne pas avoir eu de demandes des autres enseignants de l'établissement.

Pour Bénédicte, le lien reste très local, dans son cercle de connaissances personnel de l'établissement. Son RRUPN va jouer le rôle de médiateur vers les différentes ressources, formations et interlocuteurs disponibles. Ce que décrit bien Bénédicte, c'est que le RRUPN va d'abord écouter et analyser ses besoins - l'envie de réaliser des QCM pour ses élèves - avant de l'aiguiller vers telle ou telle formation. Bénédicte ne connaissait pas le dispositif auquel elle allait s'adresser, les coopératives pédagogiques numériques, avant le confinement. Déçue par les formations formelles proposées par le plan académique de formation (PAF), celle-ci voyait son horizon se limiter à un contact de confiance, le RRUPN, auquel elle s'adressait en salle des professeurs ou parfois par courriel. C'est une des caractéristiques des parcours d'appropriation ${ }^{5}$, le lien social et la dynamique collective semblent se renforcer au cours des étapes du parcours. Dans les données collectées sur les entretiens, le passage d'une étape à une autre sollicite systématiquement l'intervention d'un tiers, souvent un proche, un parent ou un ami dans les premières étapes pour aller vers des intervenants plus institutionnels en phase de construction (formations), mais chaque fois, il y a une fonction de médiation nécessaire. 


\section{Le déconfinement et la préparation de la rentrée?}

Il faut analyser ce qui s'est passé dans la période de confinement, car le télétravail et l'enseignement à distance ne s'improvisent pas, pour les enseignants comme pour tout un chacun. Et répéter, comme l'a fait le Ministre et la communication officielle de l'institution, que « tout va bien » peut avoir un effet culpabilisant chez tous ceux qui ne sont pas à l'aise dans cette situation. On a entendu dire dans les médias que les enseignants avaient « perdu » entre 5 et $8 \%$ des élèves : l'expression leur fait porter la responsabilité d'un effet qui est systémique. Nos retours du terrain décrivent qu'une partie des enseignants s'est sentie tellement délégitimée et bousculée dans ses pratiques pendant la période qu'on peut s'attendre à une épidémie de burn-out! Une autre conséquence est à craindre : celle d'une exacerbation des clivages au sein du monde enseignant. Entre les professeurs qui nont pas réussi à maintenir le lien avec leurs élèves faute de compétences numériques, ceux qui se sont repliés sur eux en rejetant le " tout numérique ", et ceux, au contraire, qui se sont révélés durant la période. Sans accompagnement, on prend le risque d'exacerber les inégalités, voire les conflits interpersonnels déjà à l'œuvre au sein du système éducatif.

\section{Quels sont les autres leviers politiques à actionner pour l'avenir du numérique éducatif?}

Il ne faut pas abuser des contre-vérités ou des slogans à la mode sur le numérique, comme on a pu le voir pendant cette crise, et avant, car ils tendent à cristalliser les résistances des uns comme l'engagement des autres. L'approche quantitative visant à dire qu'il faut « $\mathrm{X} \%$ » d'enseignement à distance dans l'Éducation nationale ou dans l'Enseignement supérieur, ne veut rien dire. Une réflexion qualitative sur les usages pédagogiques du numérique doit primer en se demandant si, et comment, on mélange présentiel et distanciel en fonction des disciplines, des objectifs, des territoires, du profil des élèves, de l'acculturation des enseignants, etc. La crise a aussi montré l'importance de valoriser et d'organiser les enseignants qui peuvent être des ressources pour leurs collègues.

\section{Conclusion}

Le confinement a placé les enseignants, les élèves et les familles dans des conditions inédites et difficiles. Nous avons pu montrer quelques exemples de pratiques pendant le confinement qui soulignent plusieurs difficultés : comment maintenir l'enseignement à distance au-delà des premières semaines ? Comment proposer un enseignement à distance qui s'adapte aux compétences et aux conditions matérielles des élèves pour éviter de creuser les inégalités ? Comment développer les pratiques nécessaires quand l'accompagnement humain sous forme de médiation est le facteur essentiel, mais ne bénéficie pas de moyens par l'institution?

Soyons clairs : l'Éducation nationale en France en tant qu'institution n'était pas du tout préparée à ce fait social total qu'a été le confinement. Le monde numérique glisse depuis quarante ans sur le monde enseignant. Malgré la succession des plans numériques, il s'articule encore mal avec l'univers scolaire. $\mathrm{Au}$-delà des difficultés matérielles, des difficultés de connexion, ce sont surtout des difficultés de pratique du numérique pédagogique qui ont été dévoilées par le confinement articulées avec des expériences préalables et des capitaux «culturels numériques " très inégaux chez les enseignants. Dépassant la question de la formation au numérique pédagogique, la question de l'accompagnement global des 
enseignants aux cultures numériques a été cristallisée par le confinement. Nous avons présenté dans cet article des témoignages d'enseignants ayant réussi, en partie, à s'adapter tout en montrant quelles sont les limites de l'enseignement à distance. Il nous manque encore ici les témoignages des enseignants en grande difficulté, ceux qui n'ont pas pu mettre en œuvre d'enseignement à distance. Enfin, d'ici la fin de l'année doivent se tenir des « états généraux du numérique éducatif », " pour faire le point sur les enseignements » de la crise selon les mots du ministre Jean-Michel Blanquer. Espérons que lors de ce rendez-vous, toutes les disciplines scientifiques qui s'intéressent au numérique auront leur mot à dire et qu’on ne réduira pas l'éducation à l'apprentissage neuro-cognitif en occultant ses contextes.

\section{Notes}

1 Opération soutenue par l'État français dans le cadre du volet e-FRAN du Programme d'investissement d'avenir (PIA 2), opéré par la Caisse des Dépôts

2 Voir le texte réglementaire en ligne: https://www.legifrance.gouv.fr/affichTexte.do? cidTexte=JORFTEXT000038129871\&dateTexte=20190727

3 En ligne : https://moodlebox.net/fr/le système utilisé par l'enseignant a été enrichi par Mélanie Auriel, Chargée de mission " collège numérique 56 " au conseil départemental du Morbihan qui propose formation, prêt et accompagnement.

4 Référent aux ressources et aux usages pédagogiques du numérique (RRUPN) : «enseignants du second degré bénéficiant d'une indemnité forfaitaire annuelle pour mission particulière. C'est la personne en charge de l'évaluation des usages numériques, des besoins en matière de formation et du conseil au chef d'établissement pour la mise en cuvre de sa politique numérique. " (Source académie de Rennes, repéré à https://www.toutatice.fr/portail/share/GUq1Bv)

5 Voir affiche : Production non anonyme - lien à ajouter après validation

\section{Références}

Cottier, P. et Burban, F. (2016). Le Lycée en régime numérique - Usages et recomposition des acteurs. Octares.

De Certeau, M. (1990). L'invention du quotidien, T.1, Arts de faire. Gallimard.

Fiévez, A. (2017). L'intégration des TIC en contexte éducatif: Modèles, réalités et enjeux. Presses de l'université du Québec.

Jouët, J. (2000). Retour critique sur la sociologie des usages. Réseaux. Communication - Technologie - Société, 18(100). 487-521; https://doi.org/10.3406/reso.2000.2235

Lapassade, G. (2016). Observation participante. Dans Jacqueline Barus-Michel (dir.) Vocabulaire de psychosociologie : Références et positions (p. 392-407). ERES. https://doi.org/10.3917/eres.barus.2016.01.0392

Larroze-Marracq, H (1999). Apprentissages scolaires et construction des connaissances: de Piaget à Vygotsky. Aprendizagem e desenvolvimento, 8(29/30), 109-119.

Levi-Strauss, C. (1990). La pensée sauvage. Paris: Plon. (Édition originale, 1962)

Plantard, P. (2014). Anthropologie des usages du numérique. Note de synthèse d'HDR, soutenue le 4 juillet 2014, Université de Nantes, Nantes. https://halshs.archives-ouvertes.fr/tel-01164360

Plantard, P. (2016). Temps numériques et contretemps pédagogiques en collège connecté. Distances et médiations des savoirs, 16 https://doi.org/10.4000/dms. 1660

Proulx, S. (2005). Penser les usages des technologies de l'information et de la communication aujourd'hui : enjeux - modèles - tendances. Dans Vieira, L. et Pinède, N. (dir.) Enjeux et usages des TIC: aspects sociaux et culturels, (Tome 1, p. 7-20). Presses universitaires de Bordeaux. 
Rabardel, P. (1995). Les hommes et les technologies. Approche cognitive des instruments contemporains, Armand Colin. hal-01017462 https://hal.archives-ouvertes.fr/hal-01017462/document

Vergnaud, G. (1998). Toward a cognitive theory of practice. Dans A. Sierpinska et J. Kilpatrick (dir.), Mathematics education as a research domain: A search for identity. (p. 227-241). Kluwer Academic Publisher.

Wasserman, S. et Faust, K. (1994). Social Network Analysis: Methods and applications. Cambridge university Press.

Wenger, E. (1998). Communities of practice. Learning, meaning and identity. Cambridge University Press.

\section{Pour citer cet article}

Perret, D. et Plantard, P. (2020). Pratiques numériques des enseignants en Bretagne pendant le confinement. Analyse anthropologique des premières données qualitatives et quantitatives. Formation et profession, 28(4 hors-série), 1-12. http://dx.doi.org/10.18162/fp.2020.686 